\title{
PRECISE, AUTOMATIC AND FAST METHOD FOR VANISHING POINT DETECTION
}

\author{
MAHZAD KALANTARI (mahzad.kalantari@ensg.eu), \\ FRANCK JUNG (Franck.Jung@ensg.eu) \\ École Nationale des Sciences Géographiques, IGN France, and IVC, Institut \\ Recherche Communications Cybernétique de Nantes (IRCCyN) UMR CNRS 6597, \\ JEANPIERRE GUEDON (jean-pierre.guedon@ @ polytech.univ-nantes.fr) \\ $\operatorname{IRCCyN}$
}

\begin{abstract}
A new approach for vanishing point detection is described. This method is based on the theorem of Thales. The main contribution of this paper is the automatic and simultaneous detection of all vanishing points of the image that consists in detecting circles in a complex cloud of points. This extraction is performed without any prior knowledge of the internal calibration. An analysis of the error propagation is done in order to give quantitative elements on the precision of the detected vanishing points.
\end{abstract}

KEYWORDS: vanishing point detection, circles detection, Thales theorem, uncertainty propagation

\section{INTRODUCTION}

AUTOMATIC detection of vanishing point constitutes a problem widely studied in computer vision for key applications ranging from robotics to camera calibration, or automatic recovery of image orientation. The $3 \mathrm{D}$ kind of information issued from the vanishing point localisation inside the image can be used in almost any natural image or video description. The human vision, or photographic images as well, present a characteristic conical geometry, transforming real world parallel lines into bundles of lines, in the recorded image, that intersect on vanishing points. These points are typical features of images from manmade objects, especially buildings, in which almost all the visible or detectable lines correspond to strictly horizontal or vertical elements. The goal of this paper is the presentation of a new algorithm allowing the automatic detection of all vanishing points generated by the segments in a 2D image space. The method presented is simple and robust. Its precision is provided by computing the standard deviation on the vanishing point localization. The model used is based on basic geometric features, working directly in the image space. Section "state of art" reviews the classical approaches. All methods start with the segment detection in the image, an aspect which is not investigated in this paper. The segment 
classifier uses either a 3D geometric domain based on the Gaussian sphere (or equivalently the Hough transform) or a 2D description from more recent works. Section "Algorithm and implementation" describes the various steps of the algorithm based on Thales theorem, and the variance analysis. Section "Assessments and Results" presents experimental results assessing the method in terms of precision and reliability of detection, and a comparison with a method that works on the unit Gaussian sphere.

\section{STATE OF ART}

Barnard (Barnard, 1983) introduced the most popular algorithm for the detection of vanishing points based on the construction of the Gaussian sphere. Its principle is to embed all vanishing lines of a $2 \mathrm{D}$ image inside a $3 \mathrm{D}$ projective space to explain the under-relationships between the scene and the projection point of view of a 2D photo. Each vanishing line intersects with the Gaussian sphere. The advantage of this method is to bring back all vanishing points in a finite space. Magee and Aggarwal (Magee, 1984) completed the approach by accumulating the projections of the intersection of the image segments on the Gaussian sphere leading to very heavy computations (in the 3D space) and very precise results. Lutton et al. (Lutton 1998) proposed a new adaptation of the Hough transform for the detection of vanishing points and sometimes later Tuytelaars (Tuytelaars, 1998) introduced an interactive method also based on the same finite space of accumulation. Shufelt (Shufelt, 1999) tries to find vanishing point on the oblique aerial images using Gaussian sphere.

The second class of methods works directly on the image space. Quan and Mohr (Quan,1989) and Den Heuvel (Den Heuvel, 1998) introduced a detection method based on geometric constraints. In 2003, Almansa (Almansa, 2003) developed a new method of vanishing point detection requiring no a priori information, but using complex probabilistic models. Brauer and Voss (Brauer, 2000) detected vanishing points in highly noisy images. F. Schaffalitzky and A. Zisserman (Schaffalitzky, 2000) extracted vanishing lines and points by planar grouping.

One can also mention Rother (Rother, 2000) who proposed a very simple method based on the intersection of the segments two by two followed by a majority vote, simple to implement but very heavy in terms of calculation time.

Our work is also based on a simple 2D geometry of the image space. It aims at a fully automatic detection of vanishing points for city buildings and street scenes. The modelling for the $3 \mathrm{D}$ scene is not required. Nonetheless, the only a priori requirement is to have enough segments in the image that are automatically detected. These segments correspond to lines, in urban images, almost totally relative to horizontal or vertical features.

\section{ALGORITHM AND IMPLEMENTATION}

The different steps of the algorithm are presented in the Fig. 1. 
Fig. 1. The different steps of the algorithm presented in this paper.

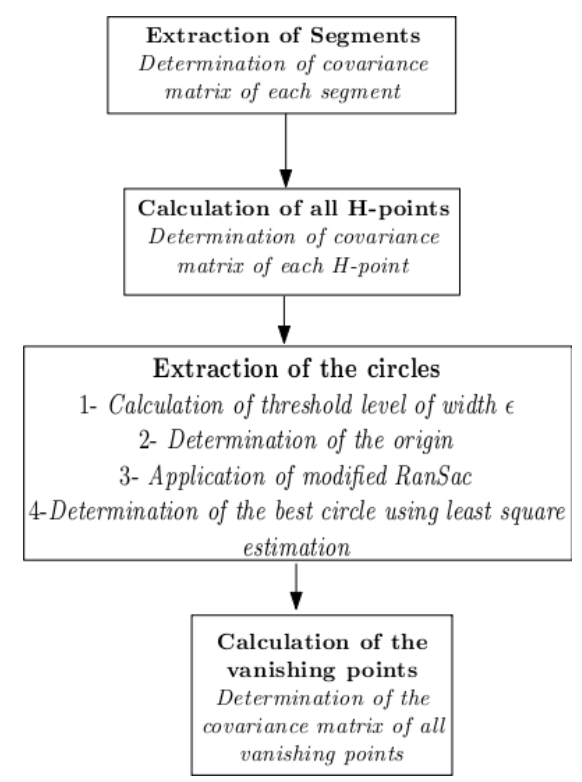

Geometry of the method.

Let $S_{1}, S_{2}, S_{3}, \ldots$ be parallel lines in the real space, their images after a conical projection are converging lines that join on the point $(P)$. This point is conventionally known as the vanishing point. Now a new bundle of lines with $O$ as center (the origin of the image) is built so that, one by one, the lines of this bundle join with a right angle the lines $S_{1}, S_{2}, S_{3}, \ldots$ on the points $H_{1}, H_{2}, H_{3}, \ldots$. According to the theorem of Thales, $H_{1}, H_{2}, H_{3}, \ldots$ and the origin $O$ define a circle (Fig. 2). 
Fig. 2. Geometric configuration based on the Thales theorem

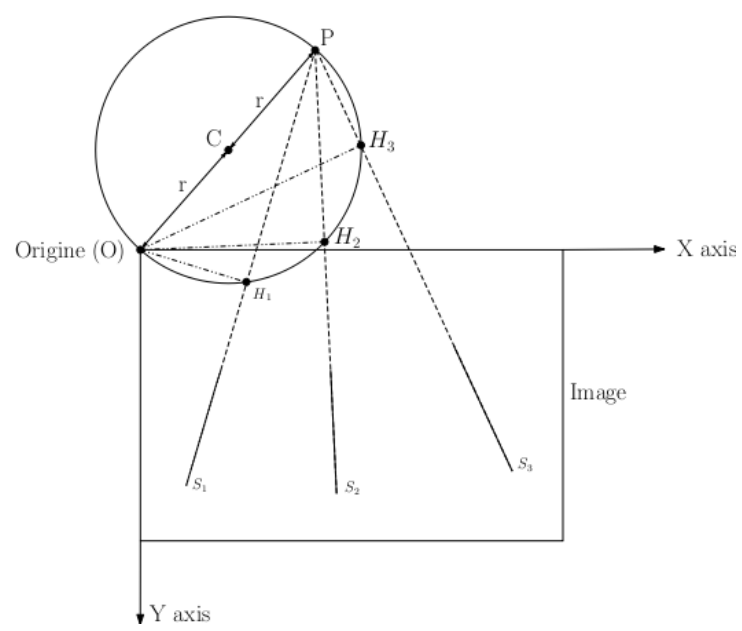

In order to determine the point $P$ (the vanishing point) it is enough to determine the parameters of the circle, which is a relatively simple operation. Therefore there are as many circles on the image as vanishing points, and thus to look for the vanishing points that correspond to the intersection of the lines carrying the segments in the images is equivalent to detect these circles (Fig. 3). The exploitation of the theorem of Thales for the research of the vanishing points has already been proposed by Brauer and Voss (Brauer, 2000), but without treating the problem put by the extraction of the mixed different circles, and without any variance analysis. The present work has therefore been completed in this sense.

Fig. 3. Example of a building, and the set of corresponding points H. One notes that the corresponding circles of the different perspective families go through the origin of the image
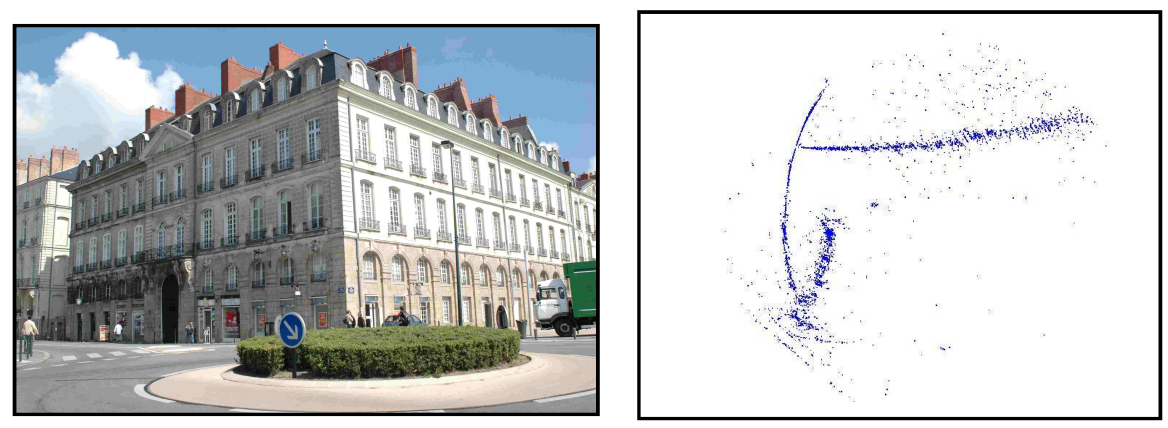

Extraction of segments.

As seen on Fig. 4, the origin $O$ has been chosen in the top left corner of the image, the $x$-axis being horizontal and the $y$-axis on the left vertical, the reference formed is not therefore of direct orientation, but every pixel receives positive coordinates. The angle $\theta$ is counted from the $x$-axis. 
Fig. 4. Definition of the image reference system

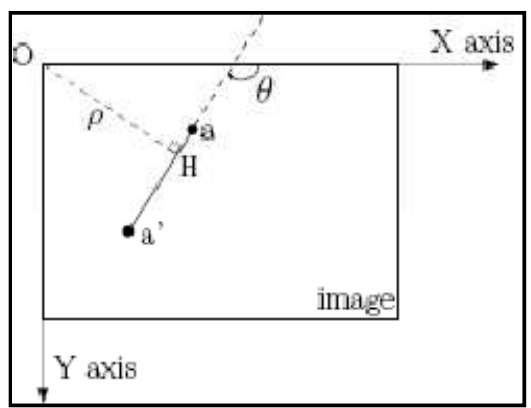

An automatic detection of the segments of the image is performed (Deriche, 1987). At this step, the covariance matrix of each segment is provided (Deriche, 1992).

Among the main results of this step, there are:

1. The extraction of all segments of the image

2. The variance-covariance matrix for each segment.

Fig. 5. Example of segments detection in an urban scene
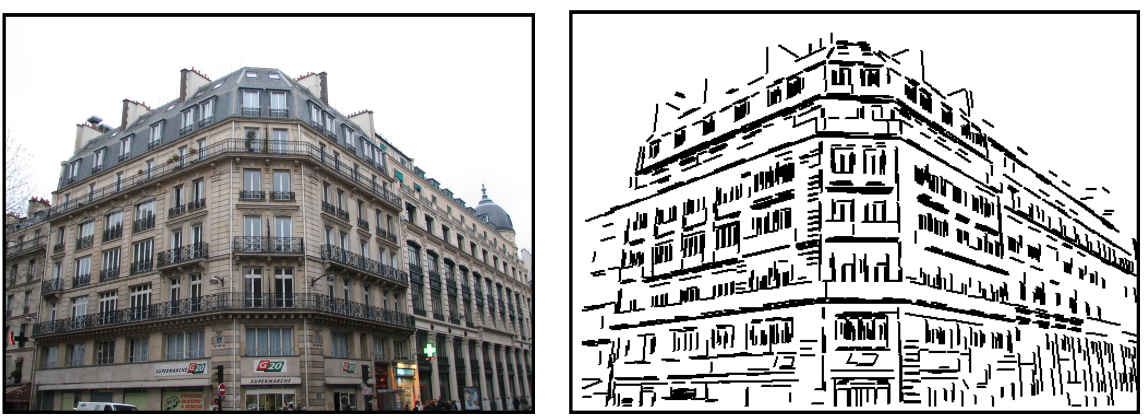

Computation of the points $H_{i}$.

The polar equation of the line, support of each detected segment, is the following :

$$
\rho=-x \sin \theta+y \cos \theta
$$

The point $H_{i}$ is defined by :

$$
H_{i}=\left(\begin{array}{c}
-\rho_{i} \cdot \sin \theta_{i} \\
\rho_{i} \cdot \cos \theta_{i}
\end{array}\right)
$$


It is important to be able to propagate the variance-covariance matrix of each segment on its corresponding point $H_{i}$. Using the general law of the propagation of variance for non linear functions, and using the Taylor theorem:

$$
\Sigma_{H_{i}}=J \times \Sigma_{\theta_{i}, \rho_{i}} \times J^{T}
$$

Where $\mathbf{J}$ is the Jacobian matrix that contains the derivatives of $H_{i}$ according to $\rho_{i}$ and $\theta_{i}$. The variance-covariance matrix $\Sigma_{H_{i}}$ on every point $H_{i}$ is then:

$$
\begin{gathered}
\Sigma_{H_{i}}=\left(\begin{array}{cc}
-\rho_{i} \cos \theta_{i} & -\sin \theta_{i} \\
-\rho_{i} \sin \theta_{i} & \cos \theta_{i}
\end{array}\right) \times\left(\begin{array}{cc}
\sigma_{\theta_{i}}^{2} & \sigma_{\rho_{i} \theta_{i}} \\
\sigma_{\rho_{i} \theta_{i}} & \sigma_{\rho_{i}}^{2}
\end{array}\right) \times\left(\begin{array}{cc}
-\rho_{i} \cos \theta_{i} & -\sin \theta_{i} \\
-\rho_{i} \sin \theta_{i} & \cos \theta_{i}
\end{array}\right)^{T} \text {, so that } \\
\Sigma_{H_{i}}=\left(\begin{array}{cc}
\sigma_{H_{i, x}}^{2} & \sigma_{H_{i, x}} \sigma_{H_{i, y}} \\
\sigma_{H_{i, x}} \sigma_{H_{i, y}} & \sigma_{H_{i, y}}^{2}
\end{array}\right)
\end{gathered}
$$

One can classically illustrate the variance covariance matrixes of the points $H_{i}$ by their error ellipses. The Fig. 6 represents the error ellipses obtained from the segments found in the Fig. 5 and the corresponding points $H_{i}$. 
Fig. 6. : Error ellipses (at 95\% confidence level) showing the error determination of the points $H$, depending of the efficiency of the tool of automatic extraction of the segments (from the image presented in Fig. 5).

\section{Circles extraction.}

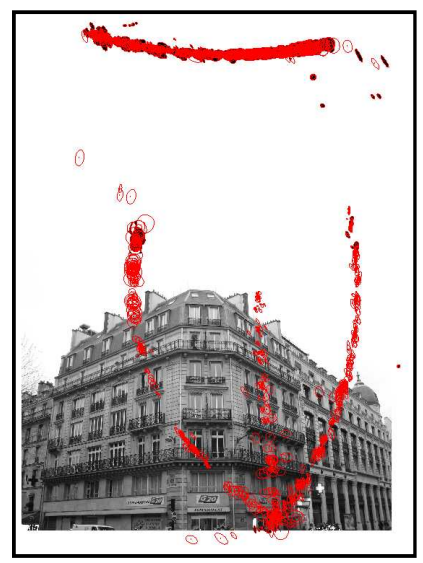

There are two distinct problems:

1 - Which model to choose for the best circle fitting a cloud of points?

2 - How to extract different circles in mixed clouds of points?

\section{Choice of the model of the best circle fitting a cloud of points.}

The different ways of modelling a circle have been reviewed; it is not useful to enter into details, as numerous references for this topic exist (Gander, 1994). A circle being defined by three points, different obvious ways exist to make a circle pass by a set of more than three points, either by minimizing the algebraic distance between the circle and the different points, and either by minimizing the geometric distance. Of course, each method has its own advantages and drawbacks.

\section{Minimization of the algebraic distance.}

Advantage of this method: it is a linear system, with a reduced calculation complexity, and therefore a reduced calculation time. Drawback: this minimization doesn't have any geometrical sense, and besides the evaluation of the parameters of the circle is not very precise.

Minimization of the geometric distance.

The advantage of this minimization is that it is more precise than the previous one (Gander, 1994). Besides, it possesses a geometrical sense. On the other hand, its inconvenience is that it implies the resolution of a non linear system, which therefore requires an approached solution. For it, we have used the previously 
described algebraic minimization. Besides, it is an iterative calculation that requires more computation power.

According to the advantages and drawbacks of each method, thereafter one or the other will be used.

Extraction of the circles in several mixed clouds of points.

The core of the algorithmic implementation of the method and its proper working relies entirely on the efficiency of this step. A method of extraction of the different circles has been inspired from the RanSac method (Fischler, 1981). Some modifications had to be brought in order to adapt it to the present situation. The different steps of the algorithm are the following:

1 - Choice of two points $H_{\mathrm{i}}$ at random from the whole set of available points $H$, since two points are sufficient to get a circle passing by the origin.

2 - Calculation of the circle passing by the sampled points and the origin, the mathematical model chosen here is the minimization of the algebraic distance.

3 - Search for all others points $H_{i}$ that are susceptible to contribute to this circle. For that it is necessary to have first fixed a capture threshold, that is to say a strip around this circle: if a point $H_{i}$ fall in this strip of width $\varepsilon$, it is kept for the definition of the circle, and otherwise it is discarded. The way of calculation of this threshold level, from the variance of the points $H_{i}$, is clarified farther.

4 - Identification of the number of selected points, re-iteration of the steps 1 , 2,3 , and finally conservation during these successive tests of the one that captured most points. This operation is reiterated many times in order to insure that the number of points captured is really maximal (here, 1000 times).

5 - At the end of this process, one keeps this set, and the corresponding captured points are then withdrawn from the initial set. Thus for the selected points, one looks for the best circle by a least squares adjustment, at that time with the help of the geometric minimization, with for approached value the parameters obtained by the calculation of the algebraic minimization. This calculation is led taking in account the uncertainties bound to every point $H_{i}$.

6 - The extraction of the circles is stopped when it remains only, for example, 5 of the initially detected points $H_{i}$.

So an efficient extraction of the circles relies entirely on the good definition of the value of the width of the capture strip $\varepsilon$. If this value is too small, a considerable number of circles without really different physical senses will be found, and on the other hand if this value is too large, one will make contribute to a given circle, and therefore to the corresponding vanishing point, points that actually should be associated to another circle.

It proves therefore to be fundamental to be able to define the capture threshold in an automatic way for a given image, and it would probably not be very adapted to use the same threshold for images with for example different resolutions. The use of a manual method to define it would cause a heavy loss of time and make lose a major interest of the method. In the optics of a complete automation of the process we did therefore try to extract parameters independent 
from the format of the image, the parameter $\varepsilon$ being bound obviously to the precision of the determination of the points $H_{i}$. In the Fig. 6 it can be seen that the error ellipses form a headband around the points $H_{i}$, and therefore the median value of the main axes of the ellipses (to $39 \%$ of confidence) can be chosen as a satisfactory threshold level for this capture using RanSaC. Therefore (i being the indication designating every point $\left.H_{i}\right), \varepsilon$ is the median of the semi-major axes of all error ellipses :

$$
\mathcal{E}=\text { median }_{i} \sqrt{\frac{1}{2}\left(\sigma_{H_{i, x}}^{2}+\sigma_{H_{i, y}}^{2}+\sqrt{\left(\sigma_{H_{i, x}}^{2}+\sigma_{H_{i, y}}^{2}\right)^{2}-4\left(\sigma_{H_{i, x}}^{2} \sigma_{H_{i, y}}^{2}-\sigma_{H_{i, x} H_{i, y}}^{2}\right)}\right)}
$$

Fig.7 . Points $\mathrm{H}_{\mathrm{i}}$ kept for a given circle of width $\varepsilon$ represented here by the zone between the two dashed curves

\section{Calculation of the best origin.}

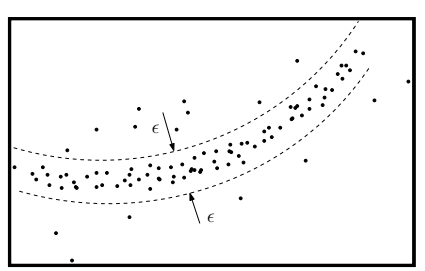

One of the key points in the algorithm is the choice of the origin. In a first time, because of simplicity, the origin can be chosen on the top left, i. e. on the origin of the image. The choice of the origin has also been discussed in the paper of (Brauer, 2000). However it was only for a given circle. The problem here it is that several circles exist and that it is necessary to choose an unique origin, optimal for all circles. Thus it is suggested to take the origin on the barycentre of the circles that have been detected when the origin is on the top left. It requires to proceed in two steps: In a first time, to detect the circles with the origin in the top left. Then in a second time, to calculate the barycentre of the circles detected and re-build the circles in this configuration. The major advantage is that the distances between the origin and the other circles are equal, so as to get the best geometric intersection between the circles. For example one may see that any fixed arbitrary origin, e. g. the middle of the image (Fig. 8), may be inappropriate in some specific situations, as it creates strong geometric difficulties to separate the circles. 
Fig. 8. : Examples of points $\mathrm{H}_{\mathrm{i}}$ for different configurations of the origin. Using the barycentre (b), the geometric intersection of the circles is optimal. a) origin in the top left. b) origin in the barycentre. c) origin in the middle of the image (unfavourable configuration)

(a)

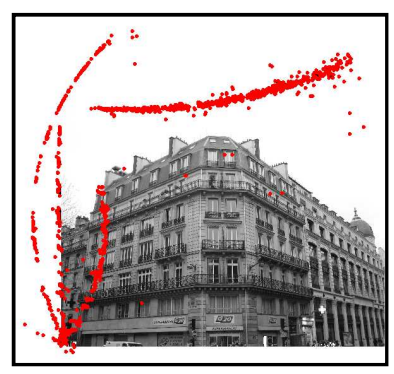

(b)

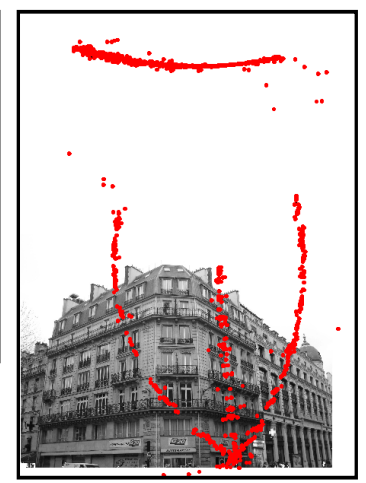

(c)

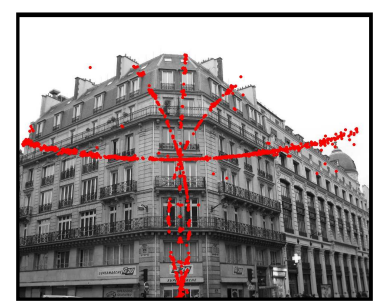

Calculation of the coordinates of the vanishing point.

Once obtained the parameters of the circle, i. e. its center coordinates $\left(X_{c}, Y_{c}\right)$ since the circle passes by the origin, the coordinates of the $P$ vanishing point are calculated very easily because $P$ is on the $O P$ diameter (Fig. 2); these coordinates are the following :

$$
\begin{aligned}
& X_{p}=2 X_{c} \\
& Y_{p}=2 Y_{c}
\end{aligned}
$$

The segments detection in the images, with the help of operators such as Canny-Dériche one, is never reliable to $100 \%$. This does not make any problem as long as one knows how to model this rate of confidence. Besides, the final goal is to be able to calculate the impact of the imprecision of the segments detected on the localization of the vanishing point. This localization will therefore be expressed accompanied of its uncertainty matrix. In this section we demonstrate how to model its impact on the vanishing point, using the uncertainty on the segment.

If we suppose that the error distribution on the segments follows a Gaussian law, the different steps of the calculation are the following (Helmert, 1872), (Cooper, 1987):

1 - Propagation of the uncertainty of the segment on the coordinates of the corresponding point $H_{i}$. 
2 - Choice of a mathematical model and definition of the observations and unknowns. The chosen mathematical model is the minimization of the geometric distance.

$$
f\left(X_{c}, X_{H}\right)=\left(\left\|\overrightarrow{X_{c}}-\overrightarrow{X_{H}}\right\|-\left\|\overrightarrow{X_{c}}\right\|\right)=0
$$

$\overrightarrow{X_{H}}$ is the vector of the points $H_{i}$, in other words the vector of the observations. In the equation (7) the model is defined for exact values of the observations, obviously fictional. For the differentiation in the following, the values $\overline{X_{c}}$ and $\overline{X_{H}}$ will be defined as the exact theoretical values.

3 - To get the exact values of the observations and unknowns, the theorem of Taylor is applied:

$$
f\left(\overline{X_{c}}, \overline{X_{H}}\right)=f\left(X_{c}{ }^{0}, X_{H}{ }^{0}\right)+\left(\frac{\partial f}{\partial X_{c}}\right)^{0} \cdot\left(\overline{X_{c}}-X_{c}{ }^{0}\right)+\left(\frac{\partial f}{\partial X_{H}}\right)^{0} \cdot\left(\overline{X_{H}}-X_{H}{ }^{0}\right)
$$

And if we note

$$
A=\left(\frac{\partial f}{\partial X_{c}}\right)^{0}, \mathrm{~B}=\left(\frac{\partial f}{\partial X_{H}}\right)^{0}, b=f\left(X_{c}^{0}, X_{H}{ }^{0}\right), x=\left(\overline{X_{c}}-X_{c}^{0}\right), v=\left(\overline{X_{H}}-X_{H}{ }^{0}\right)
$$

the formulation may be simplified in :

$b=\boldsymbol{A} x+\boldsymbol{B} v$

In other words, $\mathbf{A}$ is the Jacobian matrix related to the unknowns, i. e. the coordinates of the center of the circle, $\mathbf{B}$ is the Jacobian matrix related to the observations, i. e. the coordinates of the points $H_{i}$. However, as we have the variance covariance matrix of the points $H$, we inject this information in the equation (9). Thus the weight matrix for the observations is created, named $\mathbf{W}$. For $\mathrm{N}$ observations, the weight matrix has a dimension of $\mathrm{N} \times 2 \mathrm{~N}$. If one supposes that the points $H_{i}$ are statistically independent from each other, the matrix will have the following configuration:

$$
W=\left(\begin{array}{cccc}
\Sigma_{H_{1}}^{-1} & 0 & \ldots & 0 \\
0 & \Sigma_{H_{2}}^{-1} & \ldots & 0 \\
\ldots & \ldots & \ldots & \ldots \\
0 & 0 & \ldots & \Sigma_{H_{n}}^{-1}
\end{array}\right)
$$


$\Sigma_{H_{i}}$ is the variance covariance matrix of the points $H_{i}$ provided by the segment detector, matrix that was previously computed (equation 4).

The resolution of this system is made by the minimisation of $\sum_{i} v_{i}{ }^{T} \times W \times v_{i}$ where $v_{\mathrm{i}}$ is the vector of observations. The resolution of the system uses the Lagrange multiplier in an iterative way. At each iteration the vectors $\boldsymbol{x}$ and $\boldsymbol{v}$ are calculated. The convergence is considered as being obtained when the resulting values don't evolve anymore. The center of the circle after compensation is given by :

$$
\hat{X}_{c}=\left(A^{T}\left(B W^{-1} B^{T}\right)^{-1} A\right)^{-1} A^{T}\left(B W^{-1} B^{T}\right)^{-1} b
$$

The variance covariance matrix of the coordinates of the center of the circle is then equal to:

$$
\Sigma_{X_{c}}=\left(A^{T}\left(B W^{-1} B^{T}\right)^{-1} A\right)^{-1}
$$

And the uncertainty on the vanishing point is given by:

$$
\Sigma_{X_{p}}=\left(\begin{array}{ll}
2 & 0 \\
0 & 2
\end{array}\right) \times\left(\begin{array}{cc}
\sigma_{x_{c}}{ }^{2} & \sigma_{x_{c}, y_{c}}{ }^{2} \\
\sigma_{x_{c}, y_{c}} & \sigma_{y_{c}}{ }^{2}
\end{array}\right) \times\left(\begin{array}{ll}
2 & 0 \\
0 & 2
\end{array}\right)
$$
point $P$.

The equation 14 allows therefore to calculate the uncertainty on the vanishing

\section{ASSESSMENTS AND RESULTS}

Some examples obtained with the method presented here are shown in Fig. 9. 
Figure 9. Different examples of automatic identification of families of segments corresponding to parallels in the real space, with the superimposition of the points $H$. For a given direction (and vanishing point) extracted, the segments are presented with the same colour.
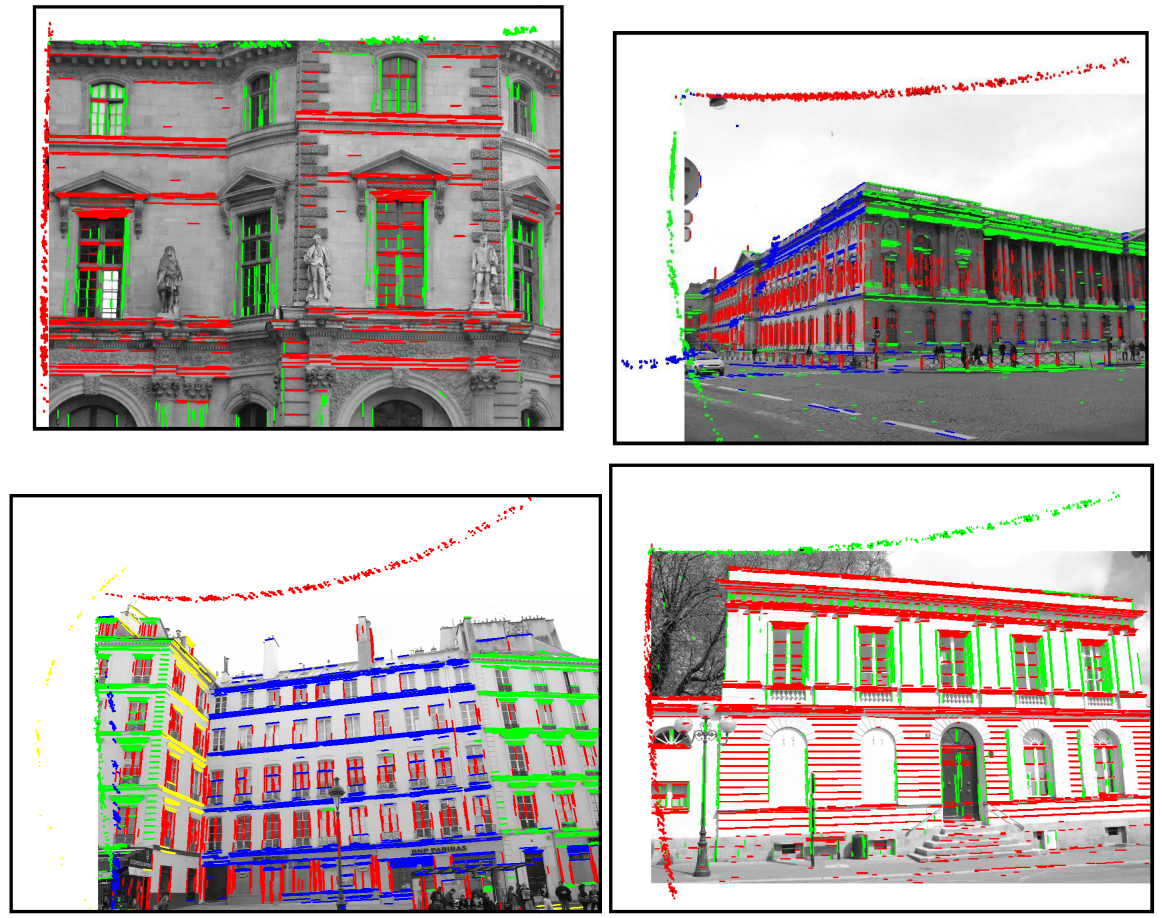

A visual validation can be obtained by automatically ortho-rectifying every facade including the vanishing point corresponding to the vertical and a vanishing point corresponding to an horizontal direction, proving that the automatic identification of the three vanishing points was correct (cf Fig. 10). This additional step can be required to automatically recognize the building facades.

Fig. 10. Based on the automatic extraction of various vanishing points, an automatic orthorectification of two facades is shown, as a simple and efficient visual test (using the image of Fig. 3).

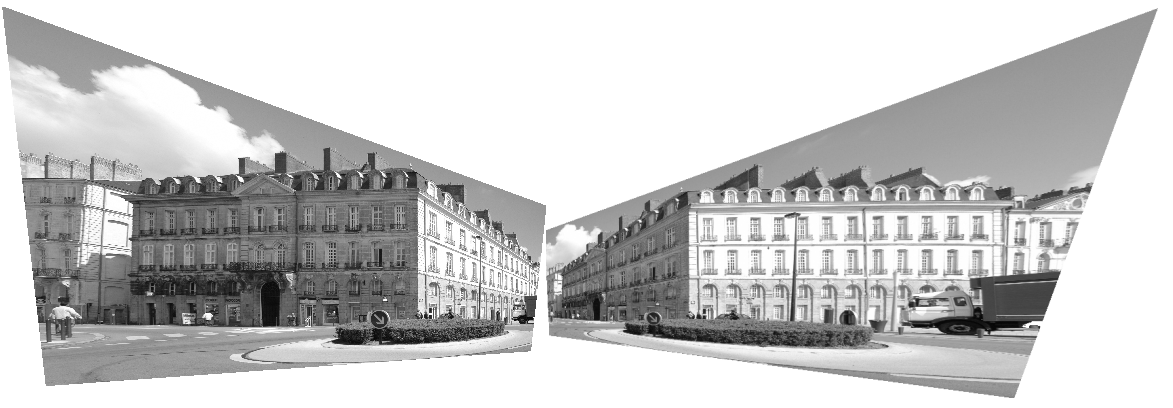




\section{Reliability of the detection of the vanishing points.}

The previous algorithms were applied to a set of different urban images, in order to identify the limits of the proposed method. To avoid lenient validations, a database has been created and is on free access on our web site http://mahzad.kalantari.free.fr/pdf.htm

On Fig. 11, some images are presented where the detection functioned satisfactorily, whereas Fig. 12 shows images where no vanishing points were provided by the algorithm. Here the results are only assessed by visual inspection of the correct number of vanishing points that must be found. These results can be considered as satisfactory.

Fig. 11.These 8 images are typical of the images where the automatic detection of vanishing points has been tested, and for which all expected points have been found

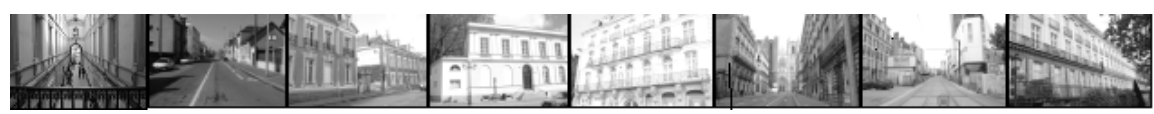

Fig. 12. These 7 images are typical of those where the automatic detection of vanishing points did not validate any point

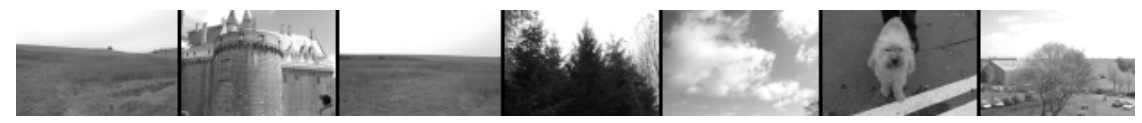

TABLE I. Statistical results on a set of 250 images

\begin{tabular}{|c|c|}
\hline $\begin{array}{c}\text { Percentage of correct detection of } \\
\text { the vertical vanishing point }\end{array}$ & $100 \%$ \\
\hline $\begin{array}{c}\text { Percentage of correct detection of } \\
\text { the horizontal vanishing point }\end{array}$ & $92 \%$ \\
\hline
\end{tabular}

The calculation part of the vanishing points without the error calculation may be performed in real time (less than 0.04 second with a processor Xeon Intel, 1.60 Ghz and 2 Go of Ram, image size 3008 x 2000). Therefore for real time applications, where in a first time it is not necessary to have the maximal precision on the vanishing point, the algorithm works properly. The error calculation part varies, with this not very powerful computer, between 0.5 to 1 minute, according to the number of segments of the image, but this part would be obviously skipped for real time, where the algorithm based on the circles works quite correctly in terms of efficiency and speed. The essential of the calculation time is due to the 
detection of the segments, as the present algorithm of assignment is comparatively extremely fast. For example for 5600 segments detected on an image of $3008 \mathrm{x}$ 2000 pixels, the algorithm (without the detection of the segments) requires 0.6 seconds without any optimization in the program.

\section{Experimental validation of the variance analysis of the extracted vanishing points.}

In order to validate the calculations bound to the extraction of the circles and the analysis of variance of the vanishing points, this method was applied on sets of theoretical segments with known vanishing points coordinates and bundles of segments and lines as shown in Fig. 13. Then a Gaussian noise with a known standard deviation was applied at the segments extremities. The robustness of the algorithm was tested on the two images (cf. Fig.13) with different segment lengths so as to estimate the effect of the length on the vanishing point accuracy. The set of operations that intervenes downstream to the process of segments extraction was then validated.

Figure 13: Theoretical segments used for checking the variance analysis of the automatically extracted vanishing points. In (13 a) the length of the segments range from 100 to 150 units (arbitrary units, the image of Fig. 13 measures 3000 x 2000 units). In (13 b) the length ranges from 1500 to 2200 units.
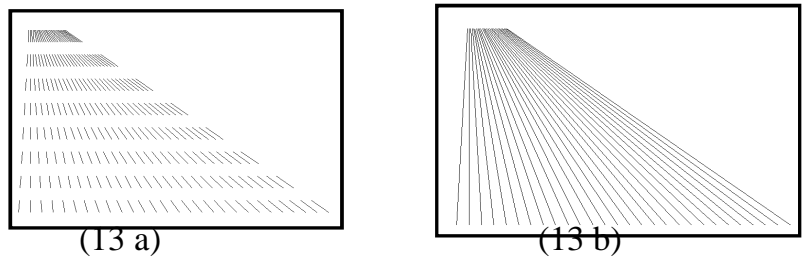

Figure 14: The computed standard deviation on the vanishing point for a few simulations on the image of figure 13 a (resp. 13 b) is shown on figure 14 a (resp. 14 b)

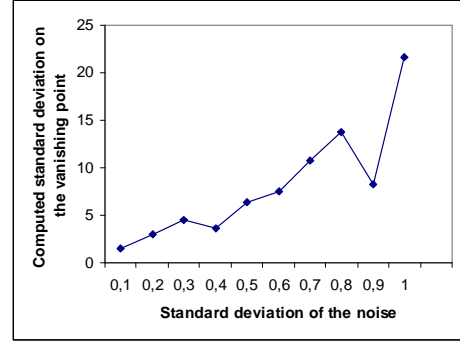

(14 a)

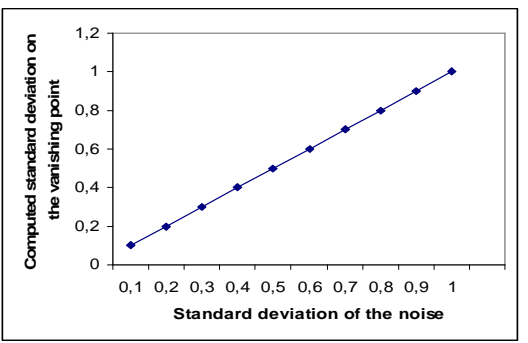

$(14 \mathrm{~b})$

Figure 14 shows the computed standard deviation on the vanishing point position as a function of the standard deviation of the noise used, for various values of this Gaussian noise (arbitrary units, the image size being $3000 \times 2000$ units, the unit thus being similar to a pixel size). These experiments demonstrate that the main sources of error in the vanishing point detection are related to the 
imperfections in the segment detection step, as well as the interest of working with long segments.

\section{Comparison with other methods of detection of vanishing points.}

It is important to be able to confront the method especially with other methods of detection of vanishing points working on the Gauss sphere, as these methods are reputed as quite efficient for the extraction of the vanishing points close to the infinity. In the examples here under, it is shown that with the method of the circles, the determination of such vanishing points is very satisfactory, and fully comparable to the methods that work in the space of the Gauss sphere.

A synthetic image was chosen with an optical axis perpendicular to the façade, so as to get vanishing points at infinity, which is considered as a quite unfavourable situation for a method working in the plane. The detection using the circles is compared with an algorithm working on the Gauss sphere (Kalantari, 2008). As it can be seen on the results, the cases where the vanishing point is at infinite create no problem to classify the different families of segments.

Fig. 15. Comparison of the method of the circles (left) with a method based on the Gauss sphere (right) in a case where the vanishing points are at infinity
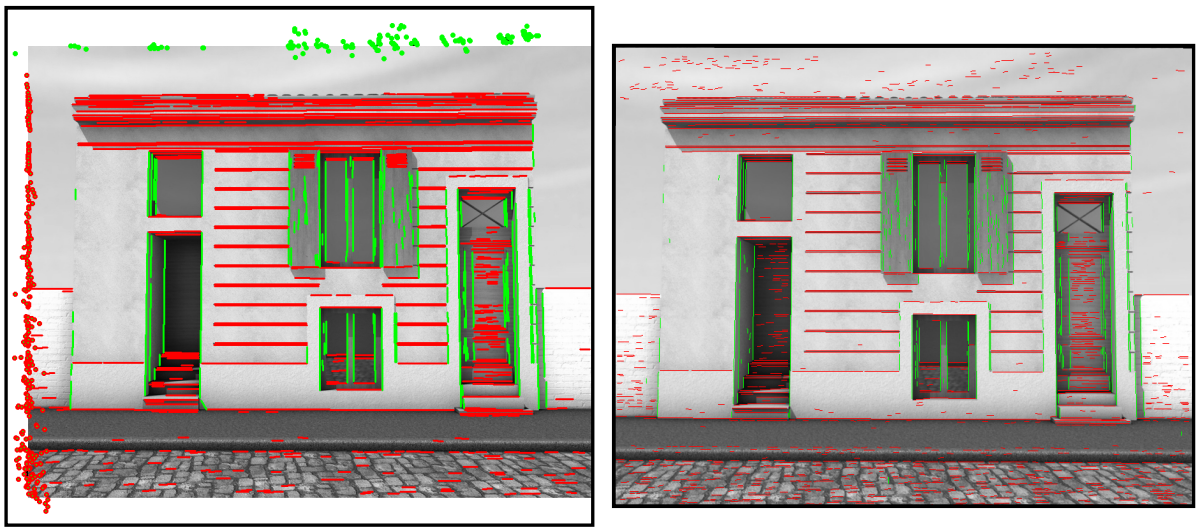

Here is another comparison on a complex real case, as the image contains 4 vanishing points, one of them being nearly at infinity (Fig. 16). 




Assessment of the impact of the distance of the vanishing point from the image.

Assessments with the help of synthesized segments have been done, so as to demonstrate the efficiency of the method even when the vanishing point is extremely far. The size of the image chosen is 3008 x 2000 pixels, and the focal distance is 3000 pixels. For each image, about one hundred segments, around 200 pixels long, are synthesized. The assessments take in account two levels of Gaussian noise, one with a standard deviation, judged as realistic, of 0.2 pixel (for the points being the extremities of each segment), and the other with a 1 pixel standard deviation, in order to value the impact of situations with very high noise. The mean values of 30 consecutive results are presented so as to facilitate the reading of the results (Fig. 17 and Fig. 18). The reference system is the same as in the Fig. 4, with the origin of the system in the top left of the image. The $\mathrm{x}$ coordinate of the vanishing point is fixed to 1504 pixels. For each new iteration, 1000 pixels are added to the y coordinate of the artificial vanishing point, that varies thus from 0 to 1000000 pixels. One can consider that for an image of 3008 x 2000 pixels, 1000000 pixels is an infinite value. The angular error is defined as the angle difference between the theoretical direction of the vanishing point and the direction of the valued vanishing point. In this simulation the method presented in this paper has been confronted to the method of the Gauss sphere (Kalantari, 2008). 
Figure 17. Results of the assessment, using the method of the circles

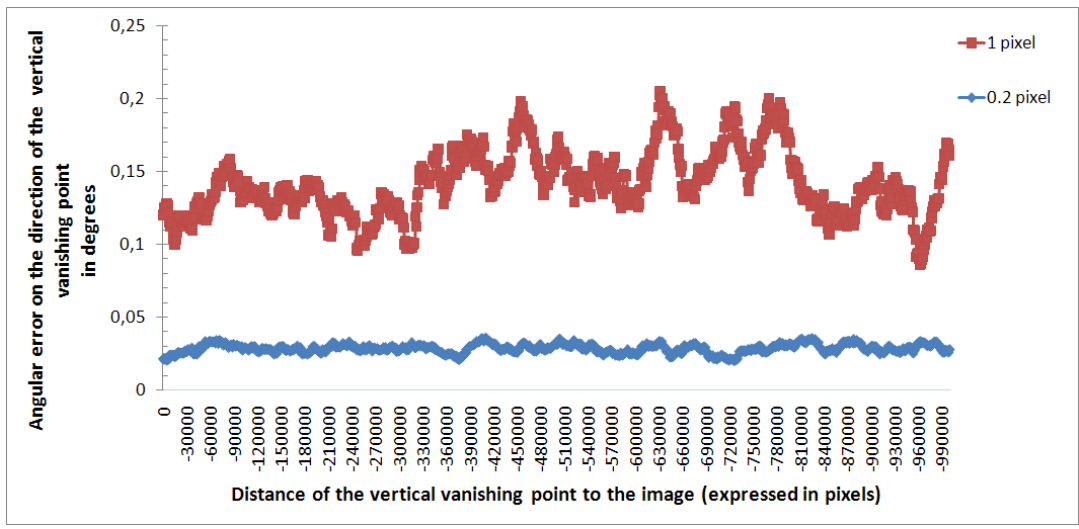

Figure 18. Results of the assessment, using the method of the Gauss sphere

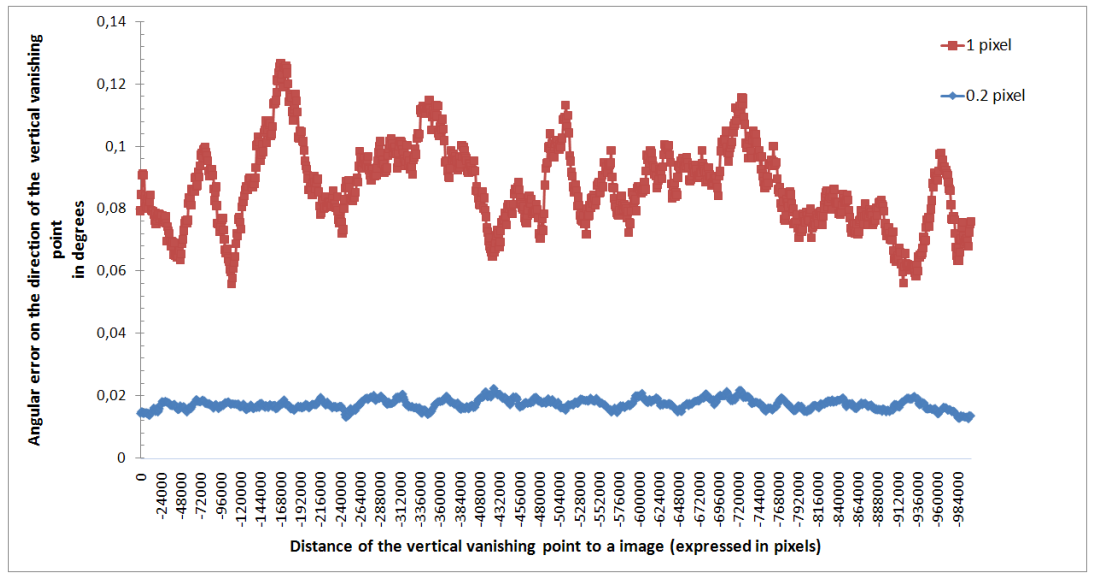

As one can see in Fig. 17 and Fig. 18, the important result is that the more or less distant localization of the vanishing point doesn't have any impact on the precision of its direction so determined, whatever the used method. In other words the method of the circles, as well as the method using the Gauss sphere (one may note that it is clearly less sensitive to the noise of the segments), operate in a satisfactory way whatever the localization of the vanishing point. The major factor that influences the determination of the localization of the vanishing point is, as anticipated, the noise on the segments. In the absence of noise the angular error is always null, showing the stability of the calculation method. One notes without surprise that for a noise of 0.2 pixel, the error found for the direction of the vanishing point is about 5 times weaker than for a noise of 1 pixel. 
For this reason it is important to have an evaluation of the localization error of the vanishing point, with the help of the error propagation presented in the section xx. This propagation allows us therefore to know accurately the impact of the segments noise on the localization of the vanishing point.

To conclude this part, it is important to note that the method of the circles, even working in the image space, doesn't meet the limitations found in methods based on the intersection of the segments on an image such as Rother's one.

\section{CONCLUSION}

For images acquired in urban environment, the presented algorithm allows to recover vanishing points entirely automatically, and to simultaneously determine their variance. One major characteristic of this algorithm is its time efficiency when compared to a Gauss sphere method. Its efficiency is comparable, even when the vanishing points are at infinity. Besides, its accuracy can still be measured. As a result, this gives a simple way to calculate the relative orientation of these images as well as a partial ortho-rectification for a real time process.

\section{ACKNOWLEDGMENT}

These works have first been led thanks to the support of the Urban Community of Le Mans, of the General Council of Sarthe and the Regional Council of the Pays de Loire, within the Ecole Supérieure des Geomètres et Topographes (ESGT) of the Conservatoire National des Arts et Métiers.

\section{REFERENCES}

Almansa, A. and Desolneux, A. and Vamech, S., 2003. Vanishing points detection without any a priori information. IEEE Trans. on PAMI, 25 (4) : 502-507.

Barnard, S., 1983. Interpreting perspective images. Artificial Intelligence, vol. 21. 1983.

Bräuer-Burchard C. and Voss, K., 2000. Robust Vanishing Point Determination in Noisy Images. International Conference on Pattern Recognition (ICPR): 1559-1562 .

Cooper, M. A. R., 1987. Control Surveys in Civil Engineering, Collins, London, 1987.

Deriche, R. and Vaillant, R. and Faugeras, O.,1992. From Noisy Edges Points to 3D Reconstruction of a Scene : A Robust Approach and Its Uncertainty Analysis . World Scientific Series in Machine Perception and Artificial Intelligence, Vol. 2: 71-79.

Deriche, R., 1987. Using Canny's criteria to derive an optimal edge detector recursively implemented, Int. J. Computer Vision, Vol. 2: 15-20.

Fischler, M. A. and Bolles, R. C., 1981. Random sample consensus: a paradigm for model fitting with applications to image analysis and automated cartography. Communications of the ACM, Vol. 24 (6), p. 381-395.

Gander, W. and Golub, G. H. and Strebel, R., 1994. Least squares fitting of circles and ellipses. $\underline{B I T}$ Numerical Mathematics. Vol. 34: 558-578.

van den Heuvel, F. A., 1998. Vanishing point detection for architectural photogrammetry. International Archives of Photogrammetry and Remote Sensing Vol. XXXII part 5: 652-659. 
Helmert, F. R., 1872. Die Ausgleichungsrechnung nach der Methode der kleinsten

Quadrate. Mit Anwendungen auf die Geodäsie, die Physik und die Theorie. Instituts zu Potsdam.

der Meßinstrumente Teubner, Leipzig.

Kalantari, M. and Jung, F. and Paparoditis, N. and Guédon, JP., 2008. Robust and Automatic Vanishing Points Detection with their Uncertainties from a Single Uncalibrated Image, by Planes Extraction on the Unit Sphere. International Archives of Photogrammetry, Remote Sensing and Spatial Information Sciences. Vol. 37 (Part 3A): 203-208.

Lutton, E. and Maitre, H. and Lopez-Krahe, J., 1994. Contribution to the determination of vanishing points using Hough transform. IEEE Trans. PAMI. Vol. 16, ํ4: 430-438.

Magee, M. J. and Aggarwal, J. K., 1984. Determining vanishing points from perspective images. CVGIP, 26 (2): 256-267.

Quan, L. and Mohr, R., 1989. Determining perspective structures using hierarchical Hough transform Pattern Recognition Letters Vol. 9: 279-286.

Rother.C., 2000 : A new approach to vanishing point detection in architectural environments. $B M V C$ : 382-391.

Schaffalitzky, F. and Zisserman, A.,2000. Planar grouping for automatic vanishing lines and points. Image and Vision Computing , 18(9): 647-658.

Shufelt, J.A., 1999. Performance evaluation and analysis of vanishing point detection, IEEE transactions PAMI, 21 (3): 282-288.

Tuytelaars, T. and Van Gool, L. and Proesmans, M. and Moons, T., 1998. The cascaded Hough transform as an aid in aerial image interpretation. Proceedings of ICCV: 67-72.

model aerial triangulation. Photogrammetric Record, 5(26): 72-81. 